\title{
Subacute Lymphocytic Thyroiditis
}

National Cancer Institute

\section{Source}

National Cancer Institute. Subacute Lymphocytic Thyroiditis. NCI Thesaurus. Code C35829.

Thyroiditis associated with painless enlargement of the thyroid gland. It occurs more frequently in females and is characterized by alterations between hyperthyroidism and hypothyroidism and the eventual return to normal thyroid gland function. 